\title{
SOME NONPROJECTIVE SUBGROUPS OF FREE TOPOLOGICAL GROUPS
}

\author{
RONALD BROWN
}

ABSTRACT. For the free topological group on an interval $[a, b]$ a family of closed, locally path-connected subgroups is given such that each group is not projective and so not free topological. Simplicial methods are used, and the test for nonprojectivity is nonfreeness of the group of path components. Similar results are given for the abelian case.

Introduction. Let $F(X)$ be the free topological group on a pointed space $X$ [4]. The object of this note is to give a family of closed subgroups of $F([a, b])$ which are not projective and so not free topological. The only previous example of a nonfree closed subgroup of an $F(X)$ has been given by Graev [4], but this was written down explicitly only in the abelian case, and I do not know if Graev's example is projective.

The structure of this paper is as follows. $\$ 1$ examines the topological group $\check{\pi}_{0} B$ of components of a topological group, and proves the key result that if $B$ is projective with open components then $\check{\pi}_{0} B$ is a discrete, free group. The methods are simple and categorical; similar results are given for the group $\pi_{0} B$ of path-components, and for the abelian case.

In $\$ 2$ the examples of nonprojective subgroups of free topological groups are given as realisations of simplicial subgroups of free simplicial groups. An Appendix summarises the definitions and results from simplicial theory which are used in $\$ 2$.

I would like to thank Dr. S. A. Morris for pointing out the problem from which this work arose, and both he and a referee for helpful comments.

1. The group of components of topological groups. For any topological space $X$, let $\breve{\pi}_{0} X$ denote its set of components with its topology as a quotient of $X$. Then $\check{\pi}_{0} X$ is totally disconnected [2, p. 125, Example 2]. Also $X$ has open components if and only if $\check{\pi}_{0} X$ is discrete-in particular this holds if $X$ is loc ally connected.

The object of this section is to describe $\check{\pi}_{0}$ for a colimit of topological groups, for free topological groups, and for projective topological groups.

Let $\mathcal{T}$ be the category of topological spaces and continuous mappings,

Received by the editors February 4, 1974 and, in revised form, July 17, 1974.

AMS (MOS) subject classifications (1970). Primary 22A05; Secondary 18G05, $55 \mathrm{~J} 10$.

Key words and phrases. Free topological group. projective topological group. 
and $\mathcal{T}_{t d c}$ the full-subcategory of $\mathcal{T}$ on the totally disconnected spaces. The corresponding categories of pointed spaces are written $\mathfrak{J}^{*}, \mathfrak{J}_{t d \mathfrak{c}}^{*}$; of topological groups and continuous morphisms are written $\mathcal{J} \mathfrak{S}_{\text {, }} \mathcal{J}_{\text {td }} \mathcal{S}_{\text {; }}$ of topological abelian groups and continuous morphisms are written $\mathcal{J} \mathscr{G}, \mathcal{T}_{t d c} \mathfrak{G} \mathcal{S}_{\text {. }}$

Proposition 1. (i) The functor $\check{\pi}_{0}$ maps $\mathcal{S} \mathcal{G}_{\text {to }} \mathcal{J}_{\text {tdc }} \bigodot_{\text {and }}$ is left ad. joint to the inclusion $\mathcal{T}_{t \mathrm{dc}} \mathrm{S} \rightarrow \mathcal{S} \mathrm{S}_{\text {. }}$

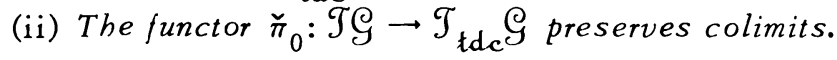

(iii) If $G=\lim _{\rightarrow} G_{a}$ is a colimit of topological groups $G_{\alpha}$ each with open components, then the topological group $\check{\pi}_{0} G$ is discrete and isomorphic as abstract group to the colimit $\lim _{\longrightarrow} \check{\pi}_{0} G_{a}$ of the discrete groups $\check{\pi}_{0} G_{a}$.

(iv) A colimit of topological groups each with open components has open components.

Proof. Here (i) is trivial, and (ii) follows from (i). (iii) follows from the fact that the colimit of discrete topological groups is discrete, and (iv) follows from (iii).

A particular case of Proposition 1 is that if $G, H$ are topological groups each with open components, then their free topological product [8] $G * H$ has open components, and $\check{\pi}_{0}(G * H) \cong \check{\pi}_{0} G * \check{\pi}_{0} H$.

We now consider free topological groups. The forgetful functor $U: \mathcal{T} \mathfrak{G}$ $\rightarrow \mathfrak{J}^{*}$ has a left-adjoint $F$, called the (Graev) free topological group functor, with universal arrow $i: X \rightarrow F(X)$ for each space with base point $X$.

Proposition 2. Let $X$ be a space with base point.

(i) The map $\check{\pi}_{0}(i): \check{\pi}_{0} X \rightarrow \check{\pi}_{0} F(X)$ is universal for continuous maps $f$ from $\check{\pi}_{0} X$ to totally disconnected topological groups such that $f$ maps the base point to the identity of the group.

(ii) If $X$ has open components, then $\check{\pi}_{0} F(X)$ is discrete and isomorphic as discrete group to $F \check{\pi}_{0}(X)$.

(iii) If $X$ has open components, so also does $F(X)$.

Proof. (i) The functor $\check{\pi}_{0} F$ is left-adjoint to the composite $\mathcal{J}_{t d c} \bigodot_{\rightarrow}$ $\mathcal{S} \stackrel{U}{\longrightarrow} \mathcal{I}^{*}$.

(ii) It follows from (i) that $\check{\pi}_{0}(i): \check{\pi}_{0} X \rightarrow \check{\pi}_{0} F(X)$ is also universal for maps from $X$ into discrete groups (since discrete implies totally disconnected). If $X$ has open components then $\check{\pi}_{0} X$ is discrete and $i: \check{\pi}_{0} X \rightarrow F \check{\pi}_{0}(X)$ is also universal for maps into discrete groups. Hence $\check{\pi}_{0} F(X) \cong F \check{\pi}_{0}(X)$.

(iii) This follows from (ii).

Corollary 3. If $X$ is a $k_{\omega}$-space with open components, then the identity component of $F(X)$ is a free topological group.

Proof. The identity component of $F(X)$ is a subgroup and is open by 
Proposition 2. The result follows from the topological Neilsen-Schreier theorem [1, Corollary 9].

Remark. The universal arrow $\check{\pi}_{0} X \rightarrow \check{\pi}_{0} F(X)$ extends to a morphism of topological groups $f: F \check{\pi}_{0}(X) \rightarrow \check{\pi}_{0} F(X)$. Proposition 2 shows that $f$ is an isomorphism if (and clearly only if) $F \check{\pi}_{0}(X)$ is totally disconnected-the latter is known to be true only in some cases, for example if $\check{\pi}_{0} X$ is 0 -dimensional and Hausdorff [7, Theorem 3.7].

We now consider projective topological groups [5]. A topological group $B$ is projective if given any diagram of morphisms of topological groups

$$
\underset{f}{\downarrow_{H}} \stackrel{B}{H}
$$

such that $f$ has a continuous section, then $\psi$ lifts to a morphism $B \rightarrow G$. Clearly a free topological group $F(X)$ is projective; I do not know of an example of a projective nonfree group.

Theorem 4. If $B$ is a projective topological group with open components, then $\check{\pi}_{0} B$ is a discrete, free group.

Proof. The identity $B \rightarrow B$ extends to a morphism $f: F(B) \rightarrow B$ which has a section the universal arrow $B \rightarrow F(B)$. Since $B$ is projective, there is a morphism $s: B \rightarrow F(B)$ such that $f s=1_{B}$. This implies that $\check{\pi}_{0} B$ is isomorphic as topological group to a subgroup of $\check{\pi}_{0} F(B)$.

Since $B$ has open components, so also does $F(B)$. Hence $\check{\pi}_{0} B$ is a discrete free group.

There are two other analogues of Theorem 4 which are worth stating. First of all, it is useful to replace $\check{\pi}_{0}$ by $\pi_{0}$, the space of path-components, with its quotient topology. There is the added complication that $\pi_{0} X$ need not be totally path-disconnected (the usual $\sin (1 / x)$ space gives an example), but Proposition 2 is still valid with connectedness notions replaced by those of path-connectedness-the proof is left to the reader.

Second, exactly similar results and proofs apply to topological abelian groups. Detailed statements and proofs are left to the reader, but for use in the next section we record an abelian, path-connectedness analogue of Theorem 4 .

Theorem 5. If $B$ is a projective topological abelian group with open path-components, then $\pi_{0} B$ is a discrete, free abelian group.

The proof is left to the reader.

2. Examples. We present our examples of nonprojective subgroups of free topological groups as realisations of simplicial subgroups of free 
simplicial groups. This has the advantage of making it immediate that the subgroups are both closed and locally path-connected. It also makes easier the computation of $\pi_{0}$. Background on simplicial theory is given in the Appendix.

Let $[a, b]$ be a closed interval of real numbers with $a$ taken as base point.

Theorem 6. Let $\epsilon$ be an integer greater than 1 , and let $B$ be the subgroup of $F([a, b])$ generated by $b$ and the elements $t, a \leq t \leq b$. Then $B$ is closed, locally path-connected and $\pi_{0} B \cong Z_{\epsilon}$. Hence $B$ is not projective.

Proof. We consider $[a, b]$ as the realisation $R(K)$ where $K$ has one nondegenerate simplex $c$ in $K_{1}$, two nondegenerate simplices $a, b$ in $K_{0}$ with $\partial_{0} c=b, \partial_{1} c=a$, and $a$ as base point. Let $C$ be the simplicial subgroup of $F(K)$ generated by $b$ and by $c^{\epsilon}$. Let $\{a, b\}$ denote also the 0 -skeleton of $K$. Then we have a commutative diagram of morphisms of simplicial groups

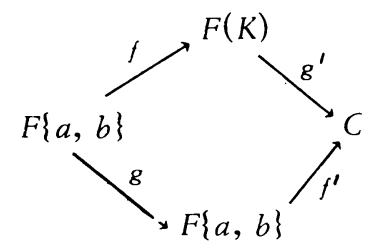

in which $f, f^{\prime}$ are inclusions, and $g, g^{\prime}$ are defined respectively by $g(b)=$ $b^{\epsilon}, g^{\prime}(c)=c^{\epsilon}$. It is straightforward to verify that this diagram is a pushout of simplicial groups. Since $\pi_{0}$ preserves colimits, we have on taking $\pi_{0}$ a pushout of groups

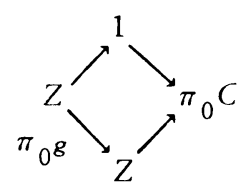

in which $\pi_{0} g$ is multiplication by $\epsilon$. Hence $\pi_{0} C \cong Z_{\epsilon}$.

Let $B$ be the realisation of $C$. Then $\pi_{0} B \cong Z_{\epsilon}$, and $B$ is a closed and locally path connected subgroup of $F[a, b]$. By Proposition A.1 of the Appen$\operatorname{dix}, B$ is generated by the realisations of the simplices $b$ and $c^{\epsilon}$, i.e. by $b$ and by $t^{\epsilon}, a \leq t \leq b$. This completes the proof.

We give another example in the nonabelian case to show how the method can be extended. First we need a theorem of Nickolas [9] to the effect that $F[a, b]$ contains $F(X)$ as closed subgroup for any compact metric space $X$ of finite dimension.

Theorem 7。 Let $\Lambda=\operatorname{gp}\left\{b_{1}, \ldots, b_{n} ; r_{1}, \ldots, r_{n}\right\}$ be the group generated by $b_{1}, \ldots, b_{n}$ with relations $r_{1}, \ldots, r_{n}$, and suppose $r_{1}, \ldots, r_{n}$ generate 
freely the subgroup $\mathrm{gP}\left\{r_{1}, \ldots, r_{n}\right\}$ of $\mathrm{gp}\left\{b_{1}, \ldots, b_{n}\right\}$. Then there is a closed subgroup $B$ of $F[a, b]$ with $\pi_{0} B$ isomorphic to $\Lambda$.

Proof. Let $K$ be the simplicial set with nondegenerate elements $a, b_{1}$, $\ldots, b_{n}$ in $K_{0}, c_{1}, \ldots, c_{n}$ in $K_{1}$ with $\partial_{0} c_{i}=a, \partial_{1} c_{i}=b_{i}, i=1, \ldots, n$.

Then the realisation $R(K)$ is the wedge of $n$ segments, and so $F R(K)$ is embeddable in $F[a, b]$ by the result of Nickolas.

Let $C$ be the simplicial subgroup of $F(K)$ generated by $b_{1}, \ldots, b_{n}$ and by the words $r_{1}^{\prime}, \ldots, r_{n}^{\prime}$ obtained from $r_{1}, \ldots, r_{n}$ by replacing each $b_{i}$ by $c_{i}, i=1, \ldots, n$. Consider the diagram of simplicial groups

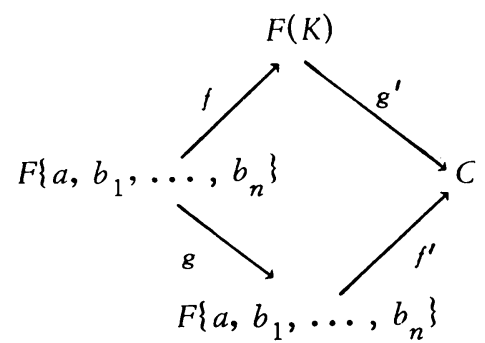

in which $f, f^{\prime}$ are inclusions, and $g, g^{\prime}$ are defined by $g\left(b_{i}\right)=r_{i}, g^{\prime}\left(c_{i}\right)=r_{i}^{\prime}$, $i=1, \ldots, n$. Certainly the diagram is commutative-I claim it is a pushout of simplicial groups. For suppose $h: F(K) \rightarrow G, k: F\left\{a, b_{1}, \ldots, b_{n}\right\} \rightarrow G$ are morphisms of simplicial groups such that $h f=k g$. It is easily checked, using the fact that $C_{1}$ is freely generated by $s_{0} b_{i}, r_{i}^{\prime}, i=1, \ldots, n$, that there is a unique morphism $\phi: C \rightarrow G$ such that $\phi g^{\prime}=h, \phi f^{\prime}=k$, given by $\phi\left(b_{i}\right)=$ $k\left(b_{i}\right), \phi\left(r_{i}^{\prime}\right)=h\left(c_{i}\right), i=1, \ldots, n$.

It follows as in the proof of Theorem 6 that $\pi_{0} C \cong \Lambda$. The group $B=$ $R(C)$ is the required closed subgroup of $F R(K)$ and so of $F[a, b]$.

In the abelian case we can obtain more complete results.

Theorem 8. Let $\Lambda$ be any finitely generated abelian group. Then there is a closed, locally path-connected subgroup $B$ of $A F[a, b]$ such that $\pi_{0} B$ is isomorphic to $\Lambda$. Hence if $\Lambda$ is not free, then $B$ is not projective.

Proof. We write $\Lambda$ as a direct sum $Z_{\epsilon_{1}}+\cdots+Z_{\epsilon_{r}}+P$ where $0<\epsilon_{i}$, $\epsilon_{i} \mid \epsilon_{i+1}$, and $P$ is a free abelian group of rank $s$.

Choose in $[a, b]$ points $a_{0}=a<a_{1}<\cdots<a_{r}<b_{1}<\cdots<b_{s}=b$. Let $K$ be the pointed simplicial set with nondegenerate elements $a_{0}, \ldots, a_{r}, b_{1}$, $\ldots, b_{s}$ in $K_{0}, c_{1}, \ldots, c_{r}$ in $K_{1}$ and with face operators given by $\partial_{0} c_{i}=$ $a_{i}, \partial_{1} c_{i}=a_{i-1}, i=1, \ldots, r$, and with $a_{0}=a$ as base point. The realisation $R(K)$ can be identified with $\left[a, a_{r}\right] \cup\left\{b_{1}, \ldots, b_{s}\right\}$, and so $R(A F(K))=A F R(K)$ can be regarded as a closed subgroup of $A F[a, b]$.

Let $C$ be the simplicial subgroup of $A F(K)$ generated by $K_{0}$ and by the elements $\epsilon_{i} c_{i}, i=1, \ldots, r$. According to $[6, \S 22] \pi_{0} C$ is isomorphic to 
$H_{0} A_{N}(C)$ where $A_{N}(C)$ is the normalised chain complex of $C$. But $A_{N}(C)$ is freely generated in dimension 0 by $a_{1}, \ldots, a_{r}, b_{1}, \ldots, b_{s}$ and in dimension 1 by $\epsilon_{1} c_{1}, \ldots, \epsilon_{r} c_{r}$ with boundary

$$
\partial\left(\epsilon_{1} c_{1}\right)=\epsilon_{1} a_{1}, \quad \partial\left(\epsilon_{i} c_{i}\right)=\epsilon_{i} a_{i}-\epsilon_{i-1} a_{i-1}, \quad i=2, \ldots, r .
$$

Since $\epsilon_{i} \mid \epsilon_{i+1}, i=1, \ldots, r$, it follows that $H_{0} A_{N}(C) \cong \Lambda$. Let $B=R(C)$. Then $\pi_{0} B$ is isomorphic to $\Lambda$. But $B$ is a closed, locally path-connected subgroup of $A F(K)$, and so of $A F[a, b]$. So the proof is complete.

It is interesting to note that $B$ is in fact the subgroup of $A F[a, b]$ generated by $a_{1}, \ldots, a_{r}, b_{1}, \ldots, b_{s}$ and by $\epsilon_{i} t, a_{i-1} \leq t \leq a_{i}, i=1, \ldots, r$.

Remarks. 1. It does not seem possible to deduce nonabelian examples from the abelian ones. The reason is that if $p: F(X) \rightarrow A F(X)$ is the natural map, and $B$ is a subgroup of $A F(X)$, then $B$ is not in general the abelianisation of $p^{-1}(B)$.

2. A similar construction to that of The orem 8 but in the nonabelian case gives a closed subgroup $B$ of $F[a, b]$ such that $\pi_{0} B \cong Z_{\epsilon_{1}} * \cdots * Z_{\epsilon_{r}}$ * $Q$, where $\epsilon_{i} \mid \epsilon_{i+1}, i=1, \ldots, r$, and $Q$ is a free group of rank $s$.

Appendix. Basic references for simplicial theory are [3], [6]-here we summarise the definitions and results we need.

Let $\Delta^{\circ}$ be the opposite category to the category $\Delta$ with objects $[\mathrm{m}]=$ $\{0, \ldots, m\}, m=0,1,2, \ldots$, and with arrows all increasing functions. If $\mathcal{C}$ is any category, a functor $X: \Delta^{\circ} \rightarrow C$ is called a simplicial object in $C$, and $X[m]$ is also written $X_{m}$. In particular we shall take for $\mathcal{C}$ the categories $\mathfrak{S}^{*}, \mathfrak{G}, \mathfrak{G} \mathcal{G}$ of countable pointed sets, countable groups, countable abelian groups respectively and so obtain the functor categories $\Delta^{\circ} \mathfrak{S}^{*}, \Delta^{\circ} \mathfrak{G}, \Delta^{\circ} \mathfrak{G}$ of pointed simplicial sets, simplicial groups and simplicial abelian groups respectively. (The countability assumption can be avoided by taking the realisation discussed below to be a functor to $k$-spaces rather than spacessee [3].)

Let $C$ be a simplicial group. A simplicial subgroup of $C$ is a simplicial group $B$ such that each $B_{m}$ is a subgroup of $C_{m}$, and the inclusion $B \rightarrow C$ is a simplicial map. We need to consider how to generate simplicial subgroups. Suppose then that $(X)_{m \geq 0}$ is a sequence such that $X_{m}$ is a subset of $C_{m}$ for $m \geq 0$. The simplicial subgroup of $C$ generated by $X$ is the smallest simplicial subgroup of $C$ containing $X$; this clearly exists and is written $\mathrm{gp} X$.

The free group, free abelian group functors $F, A F$ map $\mathfrak{f}^{*} \rightarrow \mathcal{G}, \mathcal{S}^{*} \rightarrow$ $\mathfrak{G}$ and are the left adjoints of the forgetful functors $\mathcal{G} \rightarrow \mathcal{S}^{*}, \mathfrak{G} \rightarrow \mathcal{S}^{*}$; they induce functors $F: \Delta^{\circ} \mathcal{S}^{*} \rightarrow \Delta^{\circ} \mathcal{G}, A F: \Delta^{\circ} \mathcal{S}^{*} \rightarrow \Delta^{\circ} \mathfrak{G}$ respectively. Thus if $K$ is a pointed simplicial set, then $F K(A F K)$ is the free (abelian) simplicial group on $K$.

A simplicial set is called discrete if as a functor $\Delta^{\circ} \rightarrow \mathcal{S}^{*}$ it is equiv- 
alent to a constant functor, and so can be identified with a pointed set. There is a functor $\pi_{0}: \Delta^{\circ} \mathcal{S}^{*} \rightarrow \mathcal{S}^{*}$ left-adjoint to the inclusion $\mathcal{S}^{*} \rightarrow \Delta^{\circ} \mathcal{S}^{*}$. Also $\pi_{0}$ commutes with products, and so induces functors $\Delta^{\circ} \mathfrak{G} \rightarrow \mathcal{G}, \Delta^{\circ} \mathfrak{G}$ $\rightarrow$ GY also written $\pi_{0}$ and again left-adjoint to the obvious inclusions. It follows that each $\pi_{0}$ preserves colimits, and it also follows as in $\$ 1$ that there are equivalences of functors $\pi_{0} F \simeq F \pi_{0}: \Delta^{\circ} \mathcal{S}^{*} \rightarrow \varrho_{0}, \pi_{0} A F \simeq A F \pi_{0}$ : $\Delta^{\circ} \mathfrak{S}^{*} \rightarrow \mathscr{G} \mathrm{G}_{\text {. }}$

There is a realisation functor $R: \Delta^{\circ} \mathcal{S}^{*} \rightarrow \mathcal{I}^{*}$ which has a right adjoint $S: \mathcal{T}^{*} \rightarrow \Delta^{\mathrm{o}} \mathcal{S}^{*}$, the singular functor. There is a natural bijection $\pi_{0} R(K) \rightarrow$ $\pi_{0} K$ for any simplicial set $K$. It is an important fact that if $K, L$ are simplicial sets, then the natural map $\eta: R(K \times L) \rightarrow R(K) \times R(L)$ is a homeomorphism ([6, p. 57]; it is for this reason we assume countability). It follows that if $K$ is a simplicial group with multiplication $\phi: K \times K \rightarrow K$, then $R(K)$ with multiplication

$$
R(K) \times R(K) \stackrel{\eta^{-1}}{\longrightarrow} R(K \times K) \stackrel{R(\phi)}{\longrightarrow} R(K)
$$

is a topological group. Thus $R$ induces functors $\Delta^{\circ} \mathcal{G} \rightarrow \mathfrak{T} \mathscr{Y}^{\circ} \Delta^{\circ} \mathfrak{G} \rightarrow \mathfrak{T} \mathfrak{G}$, also written $R$. Further, $R: \Delta^{\circ} \mathcal{G} \rightarrow \mathcal{T} G$ has a right adjoint the singular functor $S: \mathcal{S} \rightarrow \Delta^{\circ} \mathcal{G}$, and it may be proved from this that there are equivalences of functors $R F \simeq F R: \Delta^{\circ} \mathcal{S}^{*} \rightarrow \mathcal{J} \mathscr{G}, R A F \simeq A F R: \Delta^{\circ} \mathcal{S}^{*} \rightarrow \mathcal{T} \mathscr{G}$.

For any pointed simplicial set $K$, the realisation $R(K)$ is a $C W$-complex with cells in one-one correspondence with the nondegenerate simplices of $K$. In particular, $R(K)$ is locally path-connected. Also, by examining the nondegenerate simplices of $F(K), A F(K)$, one can obtain cell structures for $F R(K)$, $A F R(K)$.

If $L$ is a subsimplicial set of the simplicial set $K$, then $R(L)$ is a subcomplex of $R(K)$; in particular $R(L)$ is closed in $R(K)$. More generally, let $\left(X_{m}\right)_{m} \quad$ be any sequence such that $X_{m} \subseteq K_{m}, m \geq 0$. Then we define $R(X)$ to be the smallest subcomplex of $R(K)$ containing the realisations of nondegenerate simplices of $K$ which lie in $X$. The main observation we need for the explicit description of the examples of $\$ 2$ is

Proposition A.l. If $G$ is a simplicial group, and $X_{m} \subseteq G_{m}, m \geq 0$, then the subgroups $R(\mathrm{gP}(X))$ and $\mathrm{gp}(R(X))$ of $R(G)$ coincide.

Proof. Certainly these groups are both subgroups of $R(G)$.

Since $R(\mathrm{gp}(X))$ is a subgroup of $R(G)$ containing $R(X)$, it follows that $R(\mathrm{gp}(X)) \supseteq \mathrm{gp} R(X)$.

To prove that $\mathrm{gp} R(X) \supseteq R(\mathrm{gp}(X))$ we need to prove that if $H$ is any subgroup of $R(G)$ such that $H$ contains $R(X)$, then $H$ contains $R(\mathrm{gP}(X))$. For this it is sufficient to prove that if $\sigma, \tau$ are $n$-simplices of $G$ such that $R(\sigma)$ and $R(\tau)$ are contained in $H$ then so also are $R\left(\sigma^{-1}\right)$ and $R(\sigma r)$. 
That $R\left(\sigma^{-1}\right) \subseteq H$ is clear from the fact that the inverse operation in $R(G)$ is the realisation of the inverse operation in $G$. To prove $R(\sigma r) \subseteq H$, let $\hat{\sigma}, \hat{\tau}$ be the subcomplexes of $G$ generated by $\sigma, \tau$ respectively. Then $R(\sigma)=R(\hat{\sigma}), R(\tau)=R(\hat{\tau})$. So

$$
R(\sigma \tau) \subseteq R(\hat{\sigma} \hat{\tau})=R(\phi) R(\hat{\sigma} \times \hat{\tau})=R(\phi) \eta^{-1}(R(\hat{\sigma}) \times R(\hat{\tau})) .
$$

But $R(\hat{\sigma}) \subseteq H, R(\hat{\tau}) \subseteq H$, and $R(\phi) \eta^{-1}$ is the multiplication in $R(G)$. Since $H$ is a subgroup, it follows that $R(\sigma) \subseteq H$.

\section{REFERENCES}

1. R. Brown and J. P. L. Hardy, Subgroups of free topological groups and free products of topological groups, J. Lond on Math. Soc. (2) 10 (1975).

2. J. Dugundji, Topology, Allyn and Bacon, Boston, Mass., 1966. MR 33 \#1824.

3. P. Gabriel and M. Zisman, Calculus of fractions and homotopy theory, Ergebnisse der Mathematik und ihrer Grenzgebiete, Band 35, Springer-Verlag, New York, 1967. MR $35 \# 1019$.

4. M. I. Graev, Free topological groups, Izv. Akad. Nauk SSSR Ser. Mat. 12 (1948), 279-324; English transl., Amer. Math. Soc. Transl. (1) 8 (1962), 305-364. MR 10, 11 .

5. C. E. Hall, F-projective objects, Proc. Amer. Math. Soc. 26 (1970), 193-195. MR $41 \# 3555$.

6. J. P. May, Simplicial objects in algebraic topology, Van Nostrand Math. Studies, no. 11, Van Nostrand, Princeton, N. J., 1967. MR 36 \#5942.

7. S. A. Morris, Remarks on varieties of topological groups, Mat. Časopis Sloven. Akad. Vied. 24 (1974), 7.

8. A. S. Morris, E. T. Ordman and H. B. Thompson, The topology of free products of topological groups, Second Internat. Conf. on Group Theory (Canberra, 1973), Lecture Notes in Math., vol. 372, Springer, Berlin and New York, 1974, pp. 504-515.

9. P. Nickolas, Subgroups of the free topological group on $[0,1]$, J. London Math. Soc. (to appear).

SCHOOL OF MATHEMATICS AND COMPUTER SCIENCE, UNIVERSITY COLLEGE OF NORTH WALES, BANGOR, LLST 2UW, UNITED KINGDOM 